\title{
Production and spectroscopy of heavy flavor and quarkonia with the ATLAS detector
}

\author{
Constantinos Melachrinos* \\ on behalf of the ATLAS collaboration \\ University of Chicago, USA \\ E-mail: constantinos.melachrinos@cern.ch
}

\begin{abstract}
Predictions for the production of quarkonia and open heavy-flavor states at hadronic collider can now be compared to measurements performed at the LHC. ATLAS has measured the production cross section of $J / \psi$ (prompt and non-prompt) and of $\Upsilon(n S)$ (with $n=1,2,3$,), together with the inclusive production of $\mathrm{b}$-flavored hadrons. The kinematical ranges covered by the analyses extend up to values of transverse momentum significantly larger than in previous studies. The results are challenging and in some cases marginally compatible with the latest available predictions. Excited b-hadrons and quarkonia states can be detected with the ATLAS detector, opening the possibility of a broader investigation in the field of b-hadron and onia spectroscopy. The latest results, which include the observation of new particles, will be illustrated together with the perspectives for future studies.
\end{abstract}

Xth Quark Confinement and the Hadron Spectrum

8-12 October 2012

TUM Campus Garching, Munich, Germany

\footnotetext{
* Speaker.
} 


\section{Introduction}

The study of quarkonia and heavy flavor states production and spectroscopy can offer insights in the understanding of Quantum Chromodynamics. The ATLAS detector, a general-purpose detector located at the Large Hadron Collider, includes a dedicated b-physics program to study these phenomena, as will be described in Section 1.1. In particular, the study of $J / \psi$ production with the ATLAS detector will be described in Section 2, followed by the study of $\Upsilon$ production and spectroscopy in Section 3. The observation of the $\chi_{b}(3 P)$ state, the first observation of this excited state, will be discussed in Section 4, followed by the measurement of the b-hadron production cross-section in Section 5.

\subsection{The ATLAS detector}

The ATLAS detector [1] consists of tracking subdetectors that cover a pseudorapidity $(\eta)$ range up to 2.5, inside a solenoidal magnetic field of 2 Tesla, to measure the tracks of all charged particles generated by the proton-proton collisions. Liquid Argon and Tile calorimeters are subdetectors that measure energy deposits of particles, such as electrons and photons. Muon chambers cover a pseudorapidity range up to 2.7, and cover the outer part of the ATLAS detector, with a toroidal magnetic field of 0.5 Tesla. A trigger system selects events of interest to be saved for further analysis, a portion of which are dedicated b-physics streams of data, selected with dimuon samples.

\section{2. $J / \psi$ cross-section measurement}

The $J / \psi$ meson was first observed in 1974 [2], but while it is so commonly produced, the production mechanism behind it at hadron colliders is not yet understood. Using $2.2 \mathrm{pb}^{-1}$ of data from 2010, we measured the inclusive cross-section of $J / \psi$ for various rapidity bins [3], as shown in Fig. 1. We applied efficiency and acceptance corrections to take into account events not observed by the detector because of its geometry or other effects. We selected the data using a single muon trigger that required at least one muon with transverse momentum $\left(p_{T}\right)$ above $4 \mathrm{GeV}$. We then selected pairs of oppositely charged muons and plotted their invariant mass, as shown in Fig. 2. A fit to the invariant mass distribution allows us to separate the $J / \psi$ peak from combinations of muons that randomly combine in the vicinity of the $J / \psi$ invariant mass. By calculating the distance of the $J / \psi$ vertex from the primary vertex, we can further separate prompt $J / \psi$, produced from the proton-proton collision, from non-prompt $J / \psi$, produced from sequential B-meson decays. Using a fit to the "pseudoproper" time distribution, we can measure the non-prompt fraction of $J / \psi$, illustrated in Fig. 3. Our measurements agree with current measurements from the CMS experiment.

\section{3. $\Upsilon$ cross-section measurement}

In order to understand the quarkonia production mechanism and polarization, the differential and inclusive cross-section of the $\Upsilon$ meson in the (1S), (2S) and (3S) states, when the $\Upsilon$ decays to two muons [5]. This is the first measurement that includes the (2S) and (3S) states from ATLAS, 

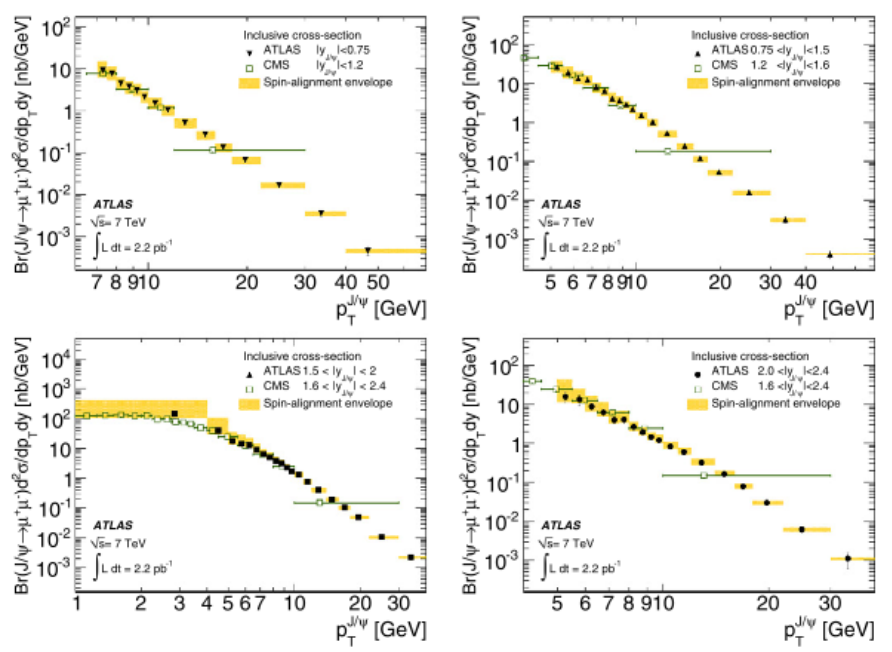

Figure 1: The measurement of the $J / \psi$ cross-section as a function of transverse momentum in various rapidity bins [3].
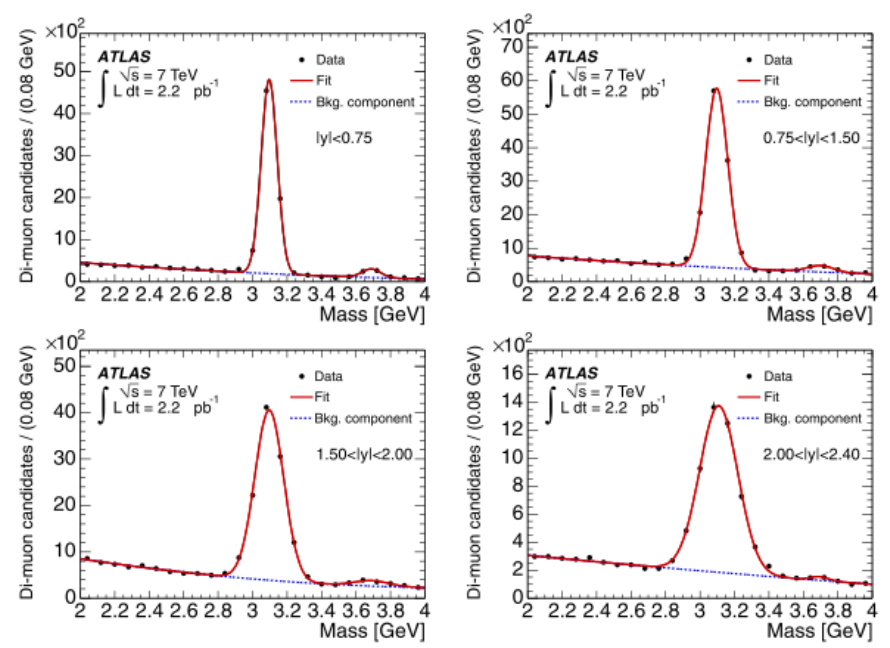

Figure 2: We used a fit to the $J / \psi$ mass distribution to extract the number of $J / \psi$ candidates in data, shown here in various rapidity bins [3].

in addition extending the transverse momentum range of the $\Upsilon$ to $70 \mathrm{GeV}$. Using $1.8 f b^{-1}$ of data from 2011, selected using a dimuon trigger with $p_{T}$ above $4 \mathrm{GeV}$, the two muons were required to have opposite charge and dimuon invariant mass between 6 and $13 \mathrm{GeV}$, for the event to be considered as a candidate event, as shown in Fig. 4.

After applying efficiency corrections per $\Upsilon$ candidate, we fit the mass spectrum to extract the weighted yield. The cross-section results for the fiducial region with muon $p_{T}$ above $4 \mathrm{GeV}$ and $|\eta|$ smaller than 2.3, were calculated and are shown in Fig. 5. Correcting for efficiencies in the full phase space, up to transverse momentum of the $\Upsilon$ of $70 \mathrm{GeV}$, we measured the inclusive crosssection for each $\Upsilon$ state, shown in Fig. 6, as well as the ratios between the different states. The ratios of the cross-sections are constant for low $p_{T}$, rising at medium $p_{T}$ and reaching a plateau 

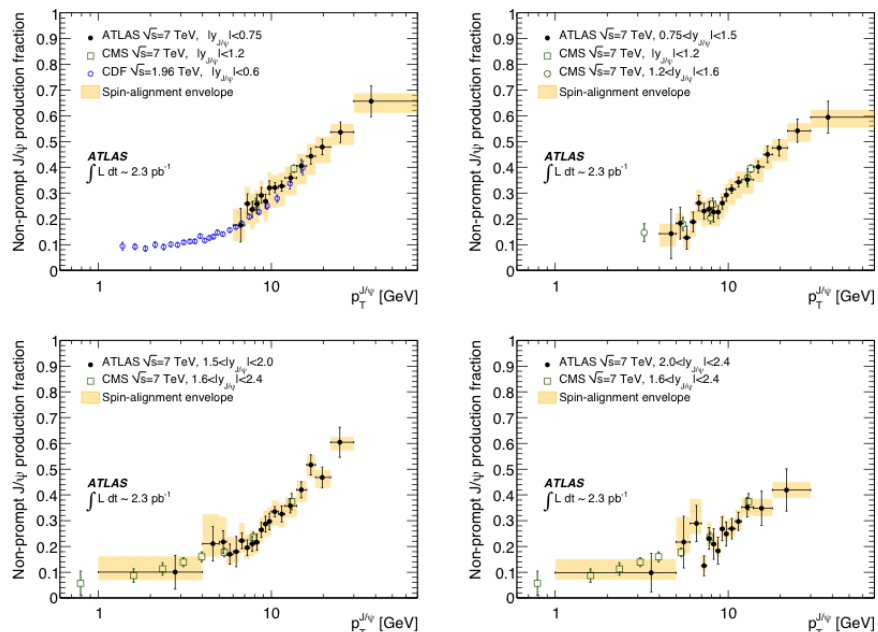

Figure 3: Performing a simultaneous fit to the $J / \psi$ mass and pseudoproper time distributions, we can extract the number of prompt and non-prompt candidates, and hence calculate the non-prompt $J / \psi$ production fraction [3].
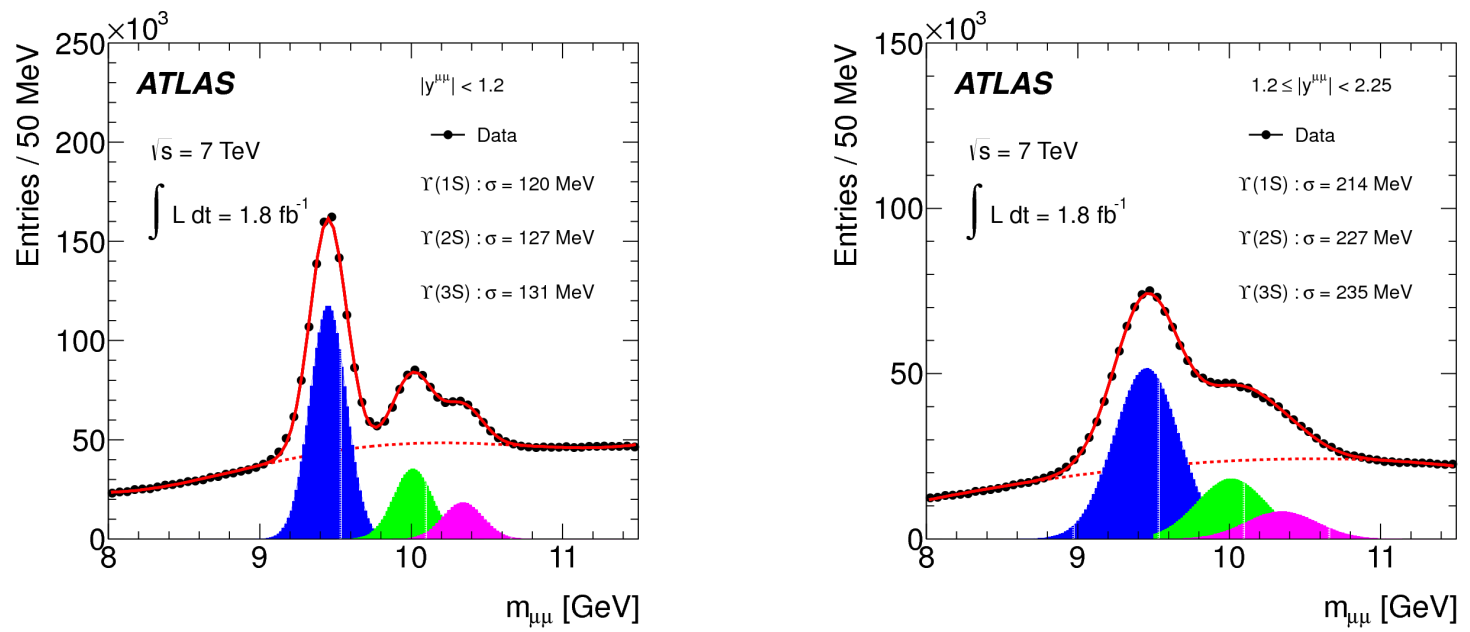

Figure 4: The $\Upsilon$ candidates, selected as oppositely charged dimuon pairs within the mass range $6-13 \mathrm{GeV}$, in two different rapidity bins [5].

at high $p_{T}$, while they are constant in rapidity, as shown in Fig. 7. Comparisons with theoretical models, the Color Evaporation and the Color Singlet models, are dominated by the spin-alignment systematic uncertainty. The integrated inclusive cross-section result for each state in the range of rapidity smaller than 2.25 and transverse momentum smaller than $70 \mathrm{GeV}$ is:

$$
\begin{aligned}
& \sigma\left(p p \rightarrow \Upsilon(1 S) \times \operatorname{Br}\left(\Upsilon \rightarrow \mu^{+} \mu^{-}\right)=8.01 \pm 0.02 \pm 0.36 \pm 0.14 n b\right. \\
& \sigma\left(p p \rightarrow \Upsilon(2 S) \times \operatorname{Br}\left(\Upsilon \rightarrow \mu^{+} \mu^{-}\right)=2.05 \pm 0.01 \pm 0.12 \pm 0.04 n b\right. \\
& \sigma\left(p p \rightarrow \Upsilon(3 S) \times \operatorname{Br}\left(\Upsilon \rightarrow \mu^{+} \mu^{-}\right)=0.92 \pm 0.01 \pm 0.07 \pm 0.02 n b\right.
\end{aligned}
$$



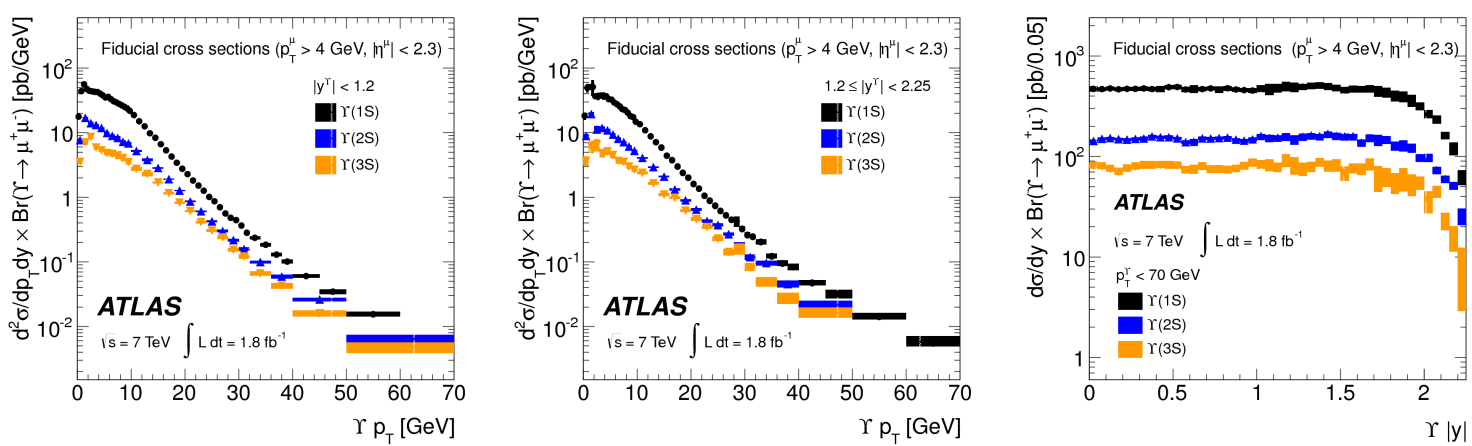

Figure 5: The $\Upsilon$ fiducial cross-section measurement as a function of transverse momentum in two rapidity bins, and as a function of rapidity [5].
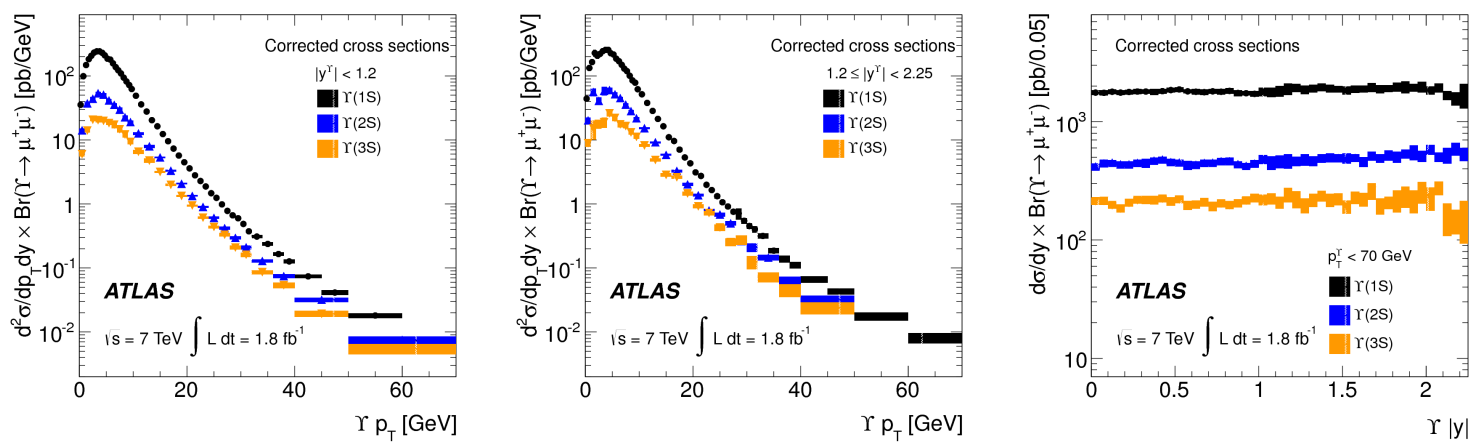

Figure 6: The $\Upsilon$ inclusive cross-section measurement as a function of transverse momentum in two rapidity bins, and as a function of rapidity in the whole phase space [5].
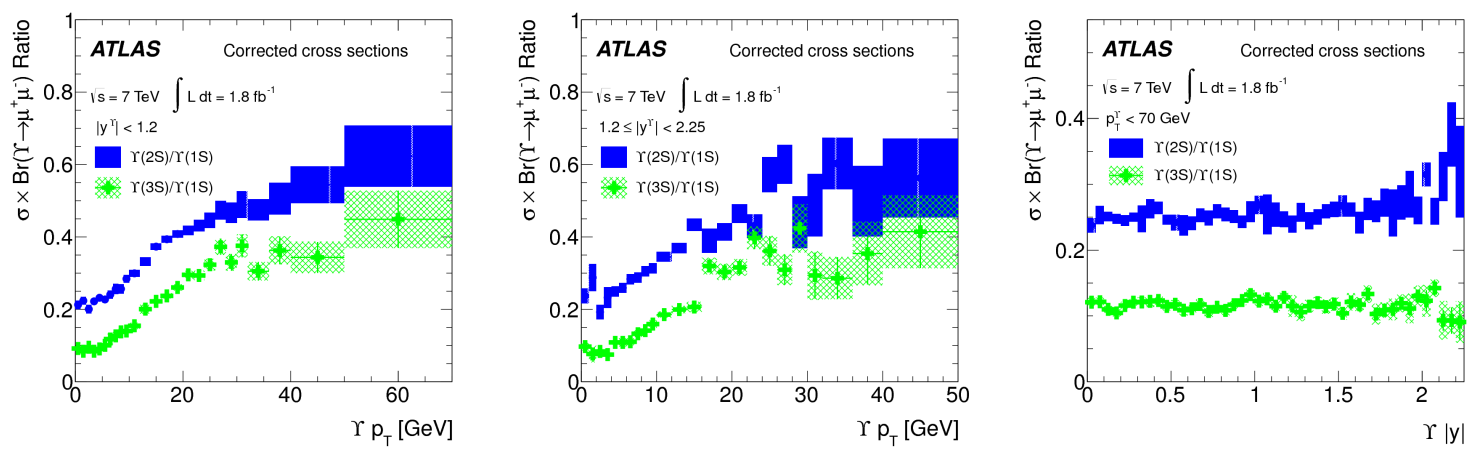

Figure 7: The ratio of the inclusive $\Upsilon$ cross-section measurement for $\Upsilon(2 S) / \Upsilon(1 S)$ and $\Upsilon(3 S) / \Upsilon(1 S)$ as a function of transverse momentum in two rapidity bins, and as a function of rapidity in the whole phase space [5].

\section{4. $\chi_{b}(3 P)$ state observation}

The observation of the $\chi_{b}(3 P)$ state was the first observation of this state at a hadron collider [6]. The $\chi_{b}(1 P)$ and $\chi_{b}(2 P)$ states had already been observed, and the $\chi_{b}(3 P)$ state was predicted to exist just below the $B-\bar{B}$ threshold. The strategy we employed was to search in radiative $\chi_{b}$ decays to $\Upsilon(1 S, 2 S) \gamma$, using both direct photon reconstruction and converted photons to 
$e^{+} e^{-}$. Using a common selection for the $\Upsilon$ candidates, we looked for oppositely charged dimuon candidates, where each muon has $p_{T}$ above $4 \mathrm{GeV}$ and the rapidity is lower than 2.3. The dimuon invariant mass was required to be between 9.25 and $9.65 \mathrm{GeV}$ for the $\Upsilon(1 S)$ candidates, and 9.8$10.1 \mathrm{GeV}$ for the $\Upsilon(2 S)$ candidates, in order to avoid interference from the $\Upsilon(3 S)$ candidates, as shown in Fig. 8. The promptly reconstructed photons were required to have transverse energy

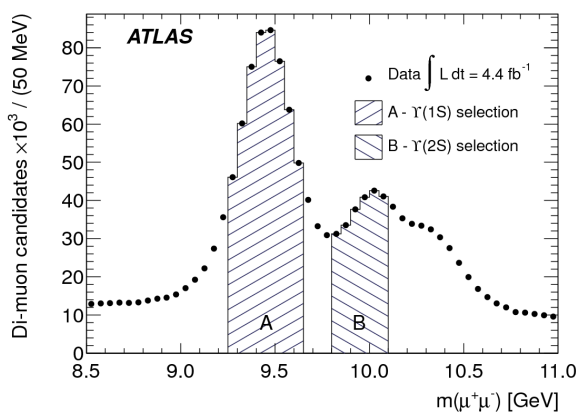

Figure 8: The $\Upsilon$ candidates, selected as oppositely charged dimuon pairs within the mass ranges 9.25-9.65 $\mathrm{GeV}(\Upsilon(1 S))$ and 9.8-10.1 GeV $(\Upsilon(2 S))$. These $\Upsilon$ candidates are combined with directly produced photons, or photons converted to electrons, to reconstruct the $\chi_{b}$ candidates. The choice of the asymmetric range for the $\Upsilon(2 S)$ candidates was taken to avoid interference with the $\Upsilon(3 S)$ candidates [6].

above $2.5 \mathrm{GeV},|\eta|$ below 2.37, and to point towards the dimuon vertex. Similarly, the converted photons were required to have $p_{T}$ above $1 \mathrm{GeV},|\eta|$ below 2.3, and the $p_{T}$ of each of the constituent electrons was required to be above $500 \mathrm{MeV}$. An extended unbinned maximum likelihood fit was performed on the $m\left(\mu^{+} \mu^{-} \gamma\right)-m\left(\mu^{+} \mu^{-}\right)+m(\Upsilon(k S))$ spectrum, in order to estimate the $\chi_{b}(3 P)$ mass barycenter, as shown in Fig. 9. The mass of the $\chi_{b}(3 P)$ state is independently mea-
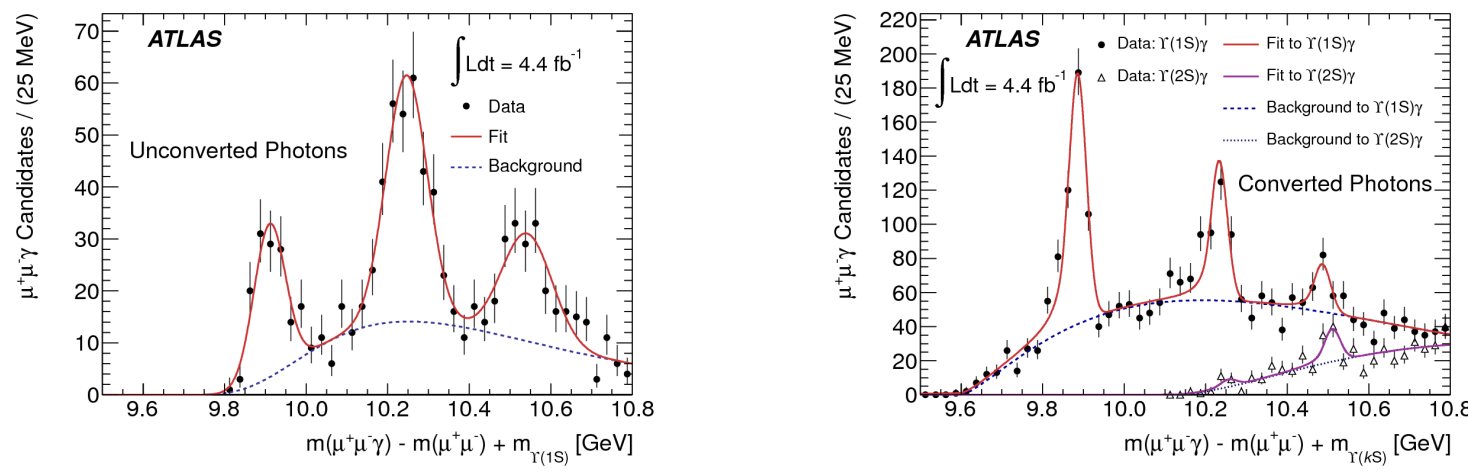

Figure 9: The $\chi_{b}(3 P)$ mass distribution, used to extract the yield of the number of $\chi_{b}(3 P)$ candidates, using directly produced photons (left) and photons converted to electrons (right) [6].

sured to be $m\left(\chi_{b}(3 P)\right)=10.541 \pm 11$ (stat) \pm 30 (syst) $\mathrm{GeV}$ for the direct photon channel, and $m\left(\chi_{b}(3 P)\right)=10.530 \pm 0.005$ (stat) \pm 0.009 (syst) $\mathrm{GeV}$ for the converted photon channel. This agrees with the theoretical prediction of $10.525 \mathrm{GeV}$ [7], and was confirmed by D0 (converted photons) [8] and LHCb (direct photons) [9]. 


\section{5. $b$-hadron production cross-section}

The measurement of the production rate of heavy quarks in hadron collisions provides a challenging test for QCD models. In addition, b-hadrons can form important backgrounds in searches for new physics, hence the understanding and measurement of the b-hadron production crosssection is important. The aim of our analysis was to measure the differential cross-section for open b-hadron production, via their semi-leptonic decay $H_{b} \rightarrow D^{*} l v X$ [10]. Using $3.3 \mathrm{pb}^{-1}$ of 2010 data, we select events that have a muon with $p_{T}$ above $6 \mathrm{GeV}$. Oppositely charged track particles with $p_{T}$ above $1 \mathrm{GeV}$ that share a common vertex are fit to form the $D^{0} \rightarrow K \pi$ candidate. The $D^{0}$ then combines with a track of opposite charge to the Kaon candidate track, with $p_{T}$ above $250 \mathrm{MeV}$, to form the $D^{*+}$ candidate, which has $p_{T}$ above $4.5 \mathrm{GeV},|\eta|$ smaller than 2.5 , and mass difference between the $K \pi$ and $D^{0}$ smaller than $40 \mathrm{MeV}$ (for $|\eta|$ smaller than 1.3) or smaller than $64 \mathrm{MeV}$ (for $|\eta|$ between 1.3 and 2.5 and $p_{T}$ of the $K^{-} \pi^{+} \pi^{+}$candidate above $12 \mathrm{GeV}$ ). As a final step, we fit the $D^{0}$ and b vertices simultaneously in the mass range $2.5 \mathrm{GeV}<m\left(D^{*} \mu\right)<5.4 \mathrm{GeV}$.

After excluding the backgrounds estimated from same sign $D^{*} \mu$ candidates and Monte Carlo simulated events, as shown in Fig. 10, we correct the measured cross-section to account for the kinematics of particle $X$, and obtain the differential cross-section distributions shown in Fig. 11. Applying acceptance corrections and taking into account the branching fraction $B R\left(H_{b} \rightarrow D^{*} \mu X\right)$, we measure the differential cross-section of $H_{b} X$ as a function of $p_{T}$ and $|\eta|$, as shown in Fig. 12.
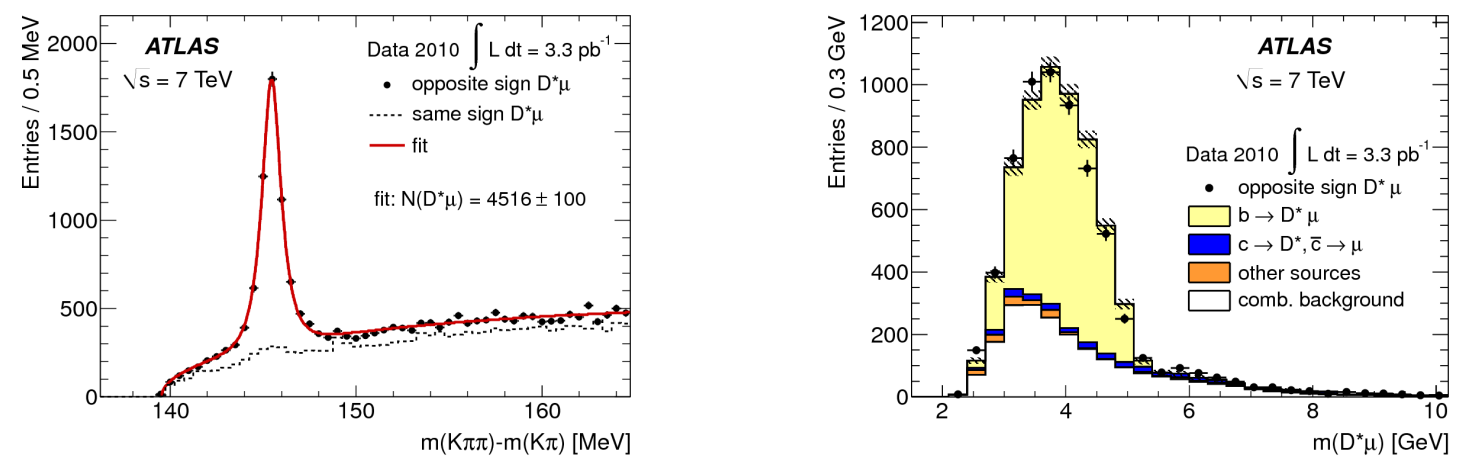

Figure 10: The mass difference $\mathrm{m}(K \pi \pi)-\mathrm{m}(K \pi)$ for $D^{*} \mu$ combinations of oppositely charged (data points) and same charge (dashed line) candidates are shown, along with a fit to estimate the number of $D^{*} \mu$ candidates (left). Without applying the invariant mass cut $2.5 \mathrm{GeV}<m\left(D^{*} \mu\right)<5.4 \mathrm{GeV}$, the various background contributions are shown (right) [10].

Extrapolating to the full phase space, the ATLAS measurement of the total cross-section is $\sigma\left(p p \rightarrow H_{b} X\right)=360 \pm 9$ (stat) \pm 34 (syst) \pm 25 (BR) \pm 12 (Lumi) \pm 77 (acceptance) $\mu b$, consistent with the LHCb measurement of $\sigma\left(p p \rightarrow H_{b} X\right)=284 \pm 20$ (stat) \pm (syst) $\mu b$ [11]. This measurement shows a slight underestimation by next-to-leading-order QCD predictions, which is however covered by the theoretical uncertainties.

\section{Acknowledgements}

This presentation was co-funded by the University of Chicago through the National Science Foundation and the Onassis Scholarships Foundation. 

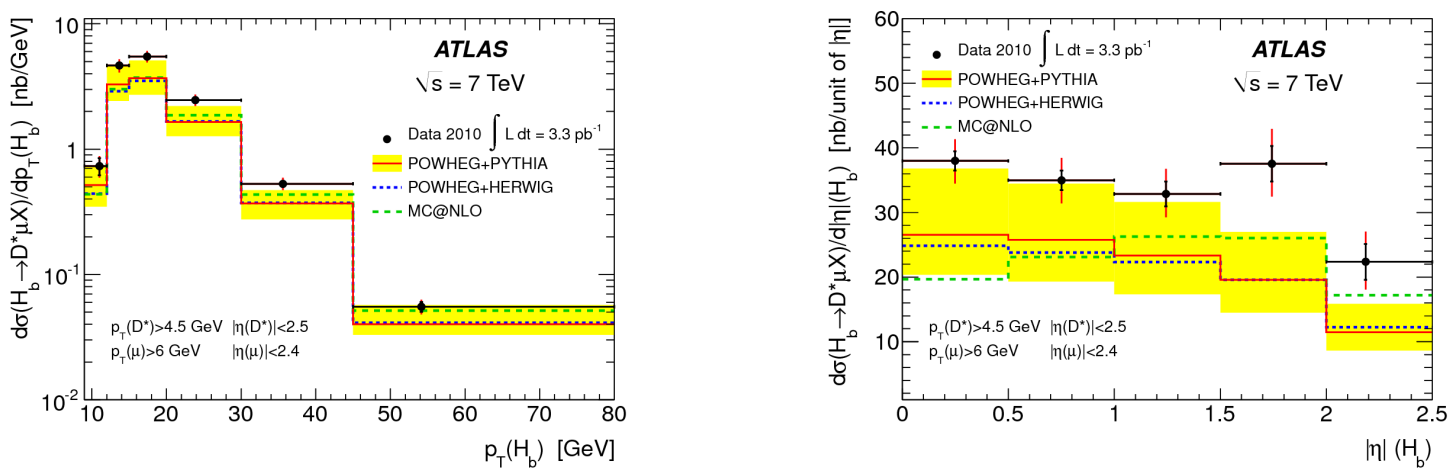

Figure 11: The differential cross-section of $H_{b} \rightarrow D^{*+} \mu^{-} X$ as a function of $p_{T}$ and $|\eta|$ in the fiducial region $p_{T}\left(D^{*+}\right)>4.5 \mathrm{GeV}, p_{T}(\mu)>6 \mathrm{GeV}, \mid \eta\left(D^{*+}\right)<2.5$ and $\mid \eta\left(\mu^{-}\right)<2.4[10]$.
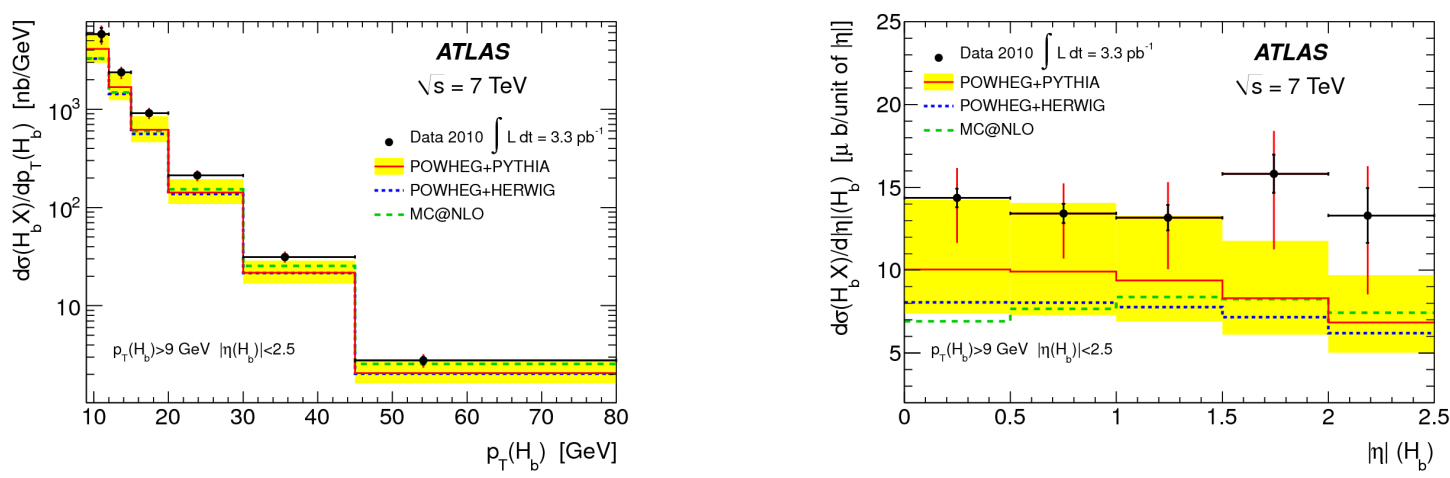

Figure 12: The differential cross-section of $H_{b} \rightarrow D^{*} \mu X$ production, after taking into account acceptance corrections and the branching fraction $B R\left(H_{b} \rightarrow D^{*} \mu X\right)$, as a function of $p_{T}$ and $|\eta|$, in the fiducial region with $p_{T}\left(H_{b}\right)>9 \mathrm{GeV}$ and $\mid \eta\left(H_{b}\right)<2.5[10]$.

\section{References}

[1] ATLAS Collaboration, JINST 3 S08003 (2008), doi:10.1088/1748-0221/3/08/S08003

[2] Aubert et al, Phys. Rev. Lett. 33, (1974) 1404-1406, doi:10.1103/PhysRevLett.33.1404 Augustin et al, Phys. Rev. Lett. 33, (1974) 1406-1408, doi:10.1103/PhysRevLett.33.1406

[3] ATLAS Collaboration, Nucl. Phys. B 850 (2011) 387-444, doi:10.1016/j.nuclphysb.2011.05.015

[4] CMS Collaboration, JHEP 02 (2012) 011, doi:10.1007/JHEP02(2012)011

[5] ATLAS Collaboration, submitted to Phys.Rev.D, arXiv:1211.7255

[6] ATLAS Collaboration, Phys. Rev. Lett. 108 (2012) 152001, doi:10.1103/PhysRevLett.108.152001

[7] L. Moytka and K. Zaleski, Eur. Phys. J. C 4, 107 (1998)

[8] D0 Collaboration, arXiv:1203.6034 (2012)

[9] LHCb Collaboration, LHCb-CONF-2012-020 (2012)

[10] ATLAS Collaboration, Nucl. Phys. B 864 (2012) 341-381, doi:10.1016/j.nuclphysb.2012.07.009

[11] LHCb Collaboration, Phys.Lett.B 694 (2010) 209-216, doi:10.1016/j.physletb.2010.10.010 